\title{
Was geschieht beim Online-Dating?
}

\section{Big Data, der Mensch und die Maschinen}

\author{
M. Spitzer, Ulm
}

Früher traf man sich in der Schule, der Uni, im Beruf oder in irgendeiner Kneipe oder Bar. Was dann geschah, wurde zwar auch bereits wissenschaftlich $\mathrm{zu}$ erfassen versucht, die Methode der unauffälligen teilnehmenden Beobachtung hatte jedoch ihre immanenten Grenzen. ${ }^{1}$ Dies hat sich mit dem Aufkommen von Computern, dem Internet und von Software, die das Begegnen zwischen den Geschlechtern online ermöglicht (Online Dating Platforms) geändert. Dort hinterlässt man Spuren, weil man meist beim Akzeptieren der Geschäftsbedingungen mit unterschreibt, dass die Daten für wissenschaftliche Zwecke weiterverwendet werden dürfen.

Diese sind im Falle des Dating im Netz deswegen so interessant, weil es sich um Abfolgen von Verhaltensweisen im sozialen Bereich handelt, die sich real abspielen. Im Gegensatz zu anderen großen Datensätzen, wo man einzelne Variablen statistisch miteinander in Verbindung bringt, liegen hier die Verbindungen bereits vor bzw. sind im entstehen, sodass man genau dies - ganz wörtlich - untersuchen kann: Das Entstehen von Verbindungen zwischen Menschen und die Abfolge von Verhaltensweisen, die hierüber letztlich entscheiden.

Die Wissenschaft vom Entscheiden ist schon älter (10), und setzt traditionell bei

1 So wurde David Buss, einem der bekanntesten Wissenschaftler auf dem Gebiet der Evolutionären Psychologie und der Psychologie von Paarung und Partnerschaft, gelegentlich sogar vorgeworfen, dass seine Erkenntnisse auf fragwürdigen Beobachtungen in "Singles Bars" beruhten (12). Aber was gab es denn vor einem viertel Jahrhundert anderes, wenn man sich bestimmte Fragen der menschlichen Annäherung empirisch zu stellen versuchte?

Nervenheilkunde 2016; 35: 653-657

Korrespondenzadresse

Prof. Dr. Dr. Manfred Spitzer, Universitätsklinikum Ulm

Klinik für Psychiatrie und Psychotherapie III

Leimgrubenweg 12, $89075 \mathrm{Ulm}$ einem rationalen Entscheider an, der Gründe vernünftig bewertet und abwägt. Dass ein solcher Rahmen für das, was sich zwischen zwei Menschen abspielt, die sich begegnen und herausfinden wollen, ob sie eine Paarbeziehung eingehen wollen - egal ob für eine Nacht oder für's Leben -, eher

\section{Dating im Netz ist deswegen so} interessant, weil man das Entstehen von Verbindungen zwischen Menschen untersuchen kann.

nicht geeignet ist, leuchtet jedem ein. Aber nicht nur deswegen, weil es bei Beziehungen zwischen Frau und Mann keineswegs immer rational zugeht, sondern weil sich solche Entscheidungen in (zuweilen sehr) kurzer Zeit abspielen, die beteiligten Personen nur eine begrenzte Zeit zum Kennenlernen haben, und zudem ihre Kapazität zur Aufnahme und Verarbeitung wesentlicher Gesichtspunkte begrenzt ist. Menschen sind endlich, und deswegen müssen sie sich dauernd entscheiden, was gleichbedeutend damit ist, dass sie nicht logisch schlussfolgern (dann wäre ja immer alles klar!). Sowohl die Daten als auch die Kapazität zu deren Verarbeitung sind im wirklichen Leben bei sehr vielen wichtigen Entscheidungen nahezu immer begrenzt - wegen unserer Endlichkeit, ganz prinzipiell.

Genau deswegen folgen Menschen bei solchen Entscheidungen keineswegs immer einem „Entscheidungsbaum“ mit all seinen Verzweigungen, sondern erledigen die Sache mittels vergleichsweise deutlich einfacheren „Heuristiken“, wie man „Daumenregeln" heute zu nennen pflegt. Wenn man sich beispielsweise zwischen sehr vielen Alternativen $\mathrm{zu}$ entscheiden hat, ist man überfordert, weil die Zahl von Einzelnem, was man unabhängig voneinander „im Kopf" behalten kann, schlicht und einfach begrenzt ist. Glaubte man noch in den 1950er-Jahren, dass diese Zahl bei $7 \pm 2$ läge (9), ${ }^{2}$ so hält man heute sogar noch weniger - nämlich $4 \pm 2$ - für die realistischere Schätzung. Das bedeutet, dass es bei Entscheidungen mit deutlich mehr als etwa

2 Zumindest wird dies seit mehr als einem halben Jahrhundert so kolportiert. Liest man das Original, so findet man eine clevere Diskussion vielfältiger psychologischer Experimente unter einem noch clevereren Titel, die damit endet, dass der Autor alles - er nennt unter anderem die sieben Meere und primären Farben, das Siebengestirn, die sieben Wochentage und die sieben Töne der Tonleiter, die sieben Todsünden und die sieben Stufen der Hölle und die sieben Zahlen, die man sich merken kann am ehesten für einen Zufall hält (9).

Abb. 1 Entscheiden als Abfolge von Entscheidungsschritten, wer herausgefiltert wird und wer nicht. Die Autoren unterscheiden die Prozesse des Suchens ( „Browsing“) und des Anschreibens ("Writing"), die jeweils dazu dienen, die Zahl der infrage kommenden Personen einzuschränken (nach 1, Fig. 1).

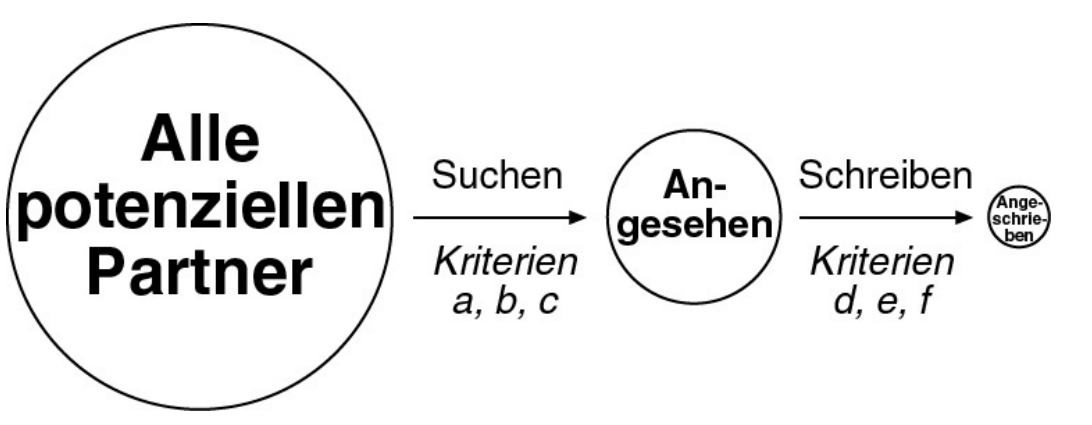


vier Alternativen sinnvoll ist, den „Entscheidungsraum " auf nicht mehr als vier Alternativen zu verkleinern. Dann hat man alles „im Kopf“ und kann entscheiden.

In der Wirtschaft spielen solche Gedanken eine große Rolle, weiß man doch schon länger, dass die Leute dann, wenn sie aus nur vier oder sechs Alternativen auswählen können, mehr kaufen als wenn sie 20 Möglichkeiten haben (11). Umfangreiche statistische Analysen eines großen Datensatzes von 1147499 Entscheidungen von 1855 zufällig ausgewählten Personen eines Online-Dating-Portals legen nahe, dass dies nicht nur im Supermarkt, sondern auch auf dem Heiratsmarkt gilt.

In Abbildung 1 ist dargestellt, wie man sich das Verhalten von Personen beim Online-Dating vorzustellen hat: Zunächst muss man unter allen überhaupt in der $\mathrm{Da}$ tenbank vorhandenen Personen diejenigen auswählen, die man sich ansehen will. Hierzu benötigt man bestimmte Kriterien, wobei in der vorliegenden Studie vor allem drei Kriterien untersucht wurden: das Alter, die Körpergröße und der Body-MassIndex (BMI). Weitere kategoreale Kriterien waren unter anderem Bildungsgrad, Fami-

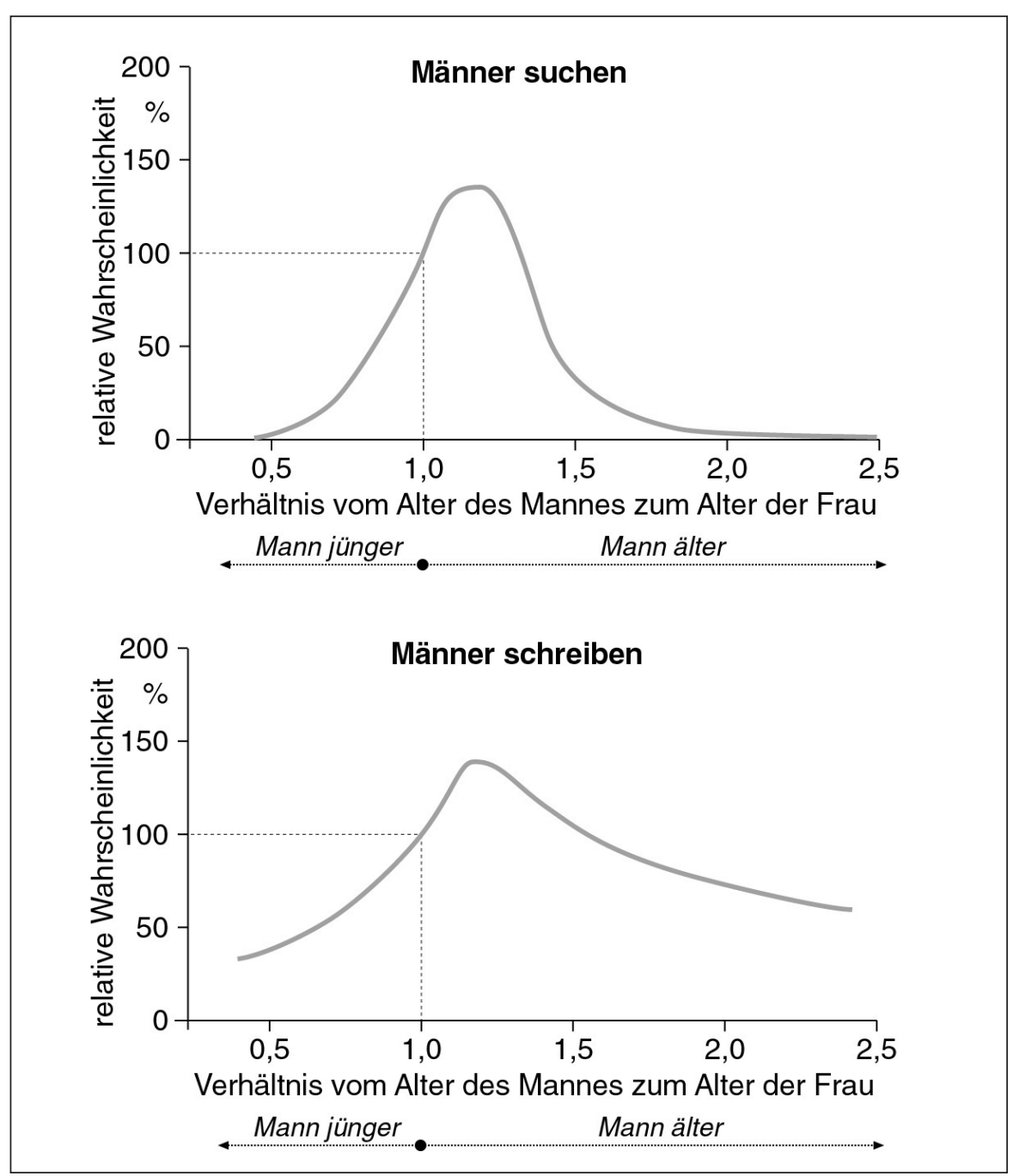

Abb. 2 Altersunterschied beim Dating. Oben:Wen Männer suchen. Relative Wahrscheinlichkeit (bezogen auf die Wahrscheinlichkeit bei gleichem Alter), in die Suche eines Mannes eingeschlossen zu werden in Abhängigkeit vom Verhältnis des Alters des (suchenden) Mannes und der (potenziellen) Partnerin. Unten: Wem Männer schreiben. Relative Wahrscheinlichkeit, dass ein Mann einer Frau schreibt in Abhängigkeit vom Verhältnis seines Alters zu dem der potenziellen Partnerin (stark schematisierte Darstellung nach 1, Fig. 3 A, B; die Daten beziehen sich auf die erwähnten 1147499 Entscheidungen, jemanden zu suchen oder anzuschreiben). lienstand, Kinder und Rauchgewohnheiten. Gemäß dieser Kriterien bildet der Nutzer des Dating-Portals zunächst eine Teilmenge der infrage kommenden potenziellen Partner. Aus dieser Teilmenge aller Teilnehmer schreibt er diejenigen aktiv (über das Dating-Portal) an, die wiederum nach Anwendung bestimmter Entscheidungskriterien infrage kommen.

\section{Menschen folgen keineswegs} immer einem verzweigten Entscheidungsbaum, sondern verwenden einfachere Daumenregeln.

Die Wissenschaftler betonen, dass es dabei nicht um Gelegenheitskontakte ging. Vielmehr untersuchte man die Mitglieder einer „established, marriage-oriented, subscription-based dating site" (1). Man kann also annehmen, dass es den Leuten - insgesamt 696 Männer und 1159 Frauen - um eine längerfristige ernsthafte Beziehung ging.

Welche Regeln bzw. Kriterien werden bei einer solchen Suche nun tatsächlich angewendet? Wie verhalten sich die Entscheider auf welcher Stufe des Entscheidungsprozesses? Verwenden sie komplexe Modelle mit gewichteten Variablen (Motto: „wenn er soundso klein ist, sollte er wenigstens soundso gebildet sein")? Oder verwenden die Nutzer ganz einfache Cut-offs (k.o.-Kriterien) wie beispielsweise „seine Größe nicht unter eins-sechzig“ oder „ihr Alter nicht über 40"? Nun machen fünf Jahre Altersunterschied bei 25-Jährigen mehr aus als bei 55-Jährigen (und bei der Größe und dem Gewicht ist es ähnlich). Um die Effekte von Alter, Größe und Gewicht daher realistisch abzubilden, wurden sie logarithmiert und die Abweichung der Ergebnisse des potenziellen Partners von den korrespondierenden logarithmierten Werten des Nutzers berechnet. Das klingt kompliziert, bewirkt aber, dass Differenzen „verhältnismäßig" bewertet werden.

Die Autoren präsentieren eine komplexe mathematische Modellierung ihrer Daten, auf die hier nicht näher eingegangen wird. Heraus kommt, ein Bild davon wie Partnerwahl online funktioniert, das datengetrieben hervorgebracht wurde und damit gegenüber methodischer Kritik („man sieht doch nur, was man sehen will“) eher immun ist als andere Verfahren. 
Zunächst einmal ergab sich, dass für das anfängliche Suchen und das spätere Anschreiben nicht immer die gleichen Kriterien eingesetzt wurden. Im Hinblick auf das Alter zeigte sich beispielsweise, dass Männer Frauen suchen (erster Schritt), die gleichaltrig oder etwas jünger sind als sie selbst ( Abb. 2 oben). Ihre Entscheidung, einer Frau zu schreiben (zweiter Schritt), hängt dagegen nicht mehr von deren Alter ab ( Abb. 2 unten).

Er sollte genau so groß sein wie Sie mit hohen Absätzen.

Den Frauen hingegen ist das Alter eines potenziellen Partners sowohl beim Suchen als auch beim Anschreiben wichtig. Passt sein Alter nicht, gerät er mit einer bis zu 400-fach geringeren Wahrscheinlichkeit überhaupt nur in ihren Suchraum! Die Autoren sprechen hier von einem „deal breaker", also einem k.o.-Kriterium, das dafür sorgt, dass bestimmte Konstellationen überhaupt keine Chance haben. ${ }^{3}$

Im Hinblick auf die Körpergröße ergab sich das gewohnte Bild, dass Frauen größere Männer und Männer kleinere Frauen bevorzugen. Dies liegt ganz einfach daran, dass Männer nun einmal größer sind als Frauen und man davon ausgehen kann, dass entsprechende Präferenzen Produkt der Evolution sind. Es machte einfach wenig Sinn, wenn Menschen das wollen, was sie prinzipiell nicht bekommen können.

Auffällig ist jedoch, wie stark der Effekt der Körpergröße auf die Präferenz gerade von Frauen ist. Eine kleinere Körpergröße des Mannes stört sie zwar beim ersten Suchen kaum (Abb. 3 oben), beim Anschreiben ist das jedoch ein k.o.-Kriterium: Einem, der kleiner ist als sie selbst, schreibt eine Frau kaum an ( $\triangle$ Abb. 3 unten). Bei Männern hingegen ist es so, dass sie sowohl beim Suchen als auch beim Anschreiben Frauen um so eher bevorzugen, je kleiner sie im Vergleich zu ihnen sind, wobei der Effekt bei einem Unterschied von mehr als etwa $15 \mathrm{~cm}$ wieder leicht abnimmt.

Weil man heute weiß, dass bei der Part-

3 Mit den Worten der Autoren: „Age differences are the biggest deal breaker. [...] women can be up to 400 times less likely to browse someone with an undesirable value of age (all else equal)“ (1).

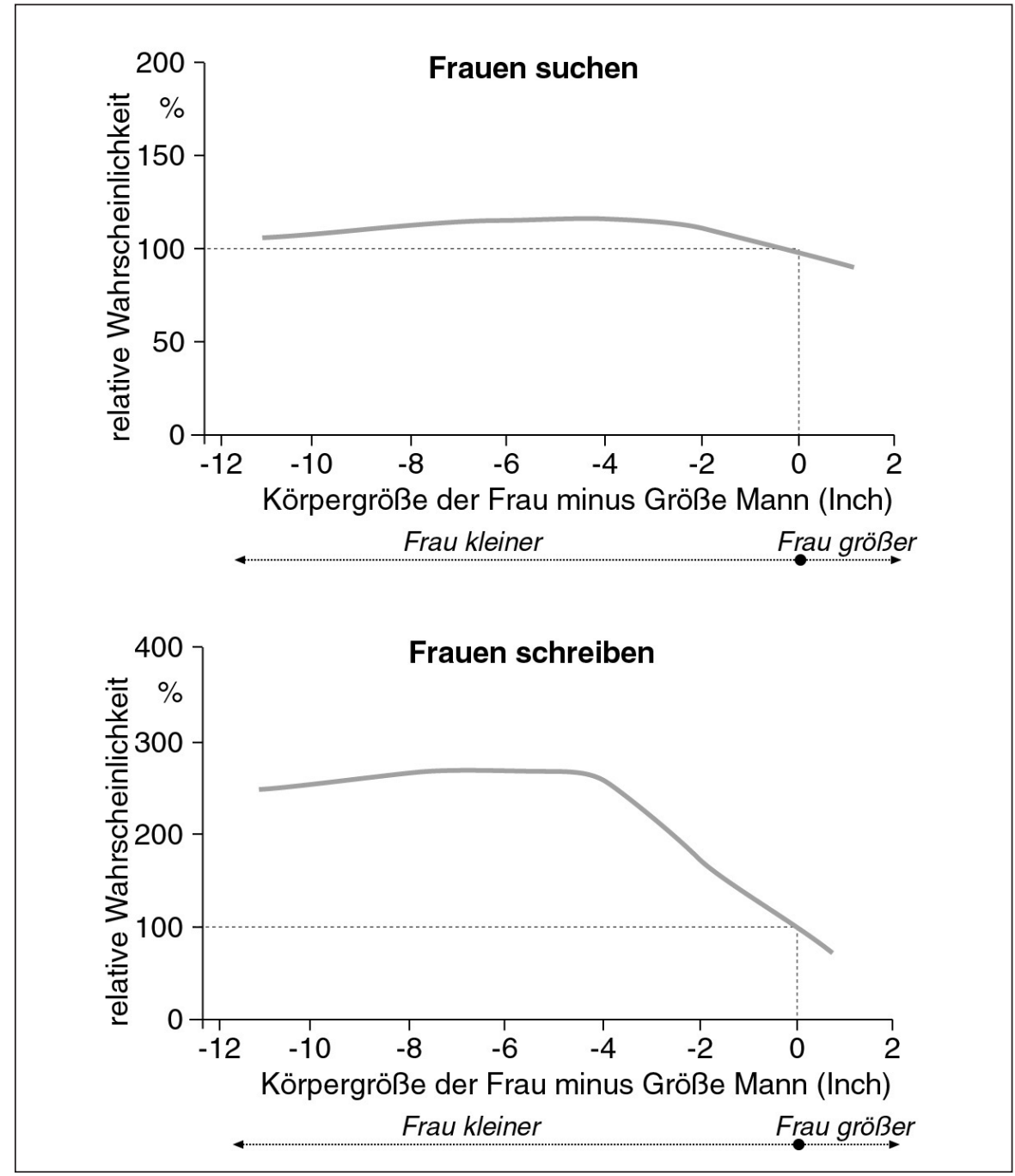

Abb. 3 Einfluss der Körpergröße beim Dating. Oben: Wen Frauen suchen. Relative Wahrscheinlichkeit (bezogen auf die Wahrscheinlichkeit bei gleicher Größe), in die Suche einer Frau eingeschlossen zu werden in Abhängigkeit vom Verhältnis der Größe der (suchenden) Frau und des (potenziellen) Partners. Unten: Wem Frauen schreiben. Relative Wahrscheinlichkeit, dass eine Frau einem Mann schreibt in Abhängigkeit vom Verhältnis ihrer Größe zu der des potenziellen Partners (stark schematisierte Darstellung nach 1, Fig. 5 C, D).

nerwahl in vielerlei Hinsicht auf Gleichheit (z. B. der Hautfarbe, der Herkunft, des Bildungsstandes, der politischen Einstellung, des Charakters, der Werte) sehr großen Wert gelegt wird, kann man die Frage stellen, warum die Paare bei der Größe auf Unterschiede aus sind. Gewiss liegen auch diese in bestimmten Grenzen - eine der vielen Regeln hierzu besagt: Er sollte genau so groß sein wie Sie mit hohen Absätzen aber dennoch sind sie da und spielen bei der Partnerwahl eine nicht geringe Rolle. Der Grund hierfür ist, dass Attribute, die mit dem Geschlecht verknüpft sind (und das ist die Größe nun einmal) auch mit den geschlechtsspezifischen Eigenschaften, die ja gesucht werden, in Verbindung stehen. Frauen sind kleiner und schwächer als Männer, und kleinere schwächere Frauen sind damit „weiblicher“. Bei Männern ist es genau umgekehrt. Und weil Männer eine „Frau“ suchen und Frauen einen „Mann“, sind die Boulevardblätter - obwohl es mittlerweile auch Damenfußball und männliche Kleiderständer gibt - voll von Beziehungen zwischen zierlichen Modells und muskulösen Fußballern.

Auch beim Körpergewicht (BMI) finden sich Unterschiede der Entscheidungskriterien in Abhängigkeit von Geschlecht 

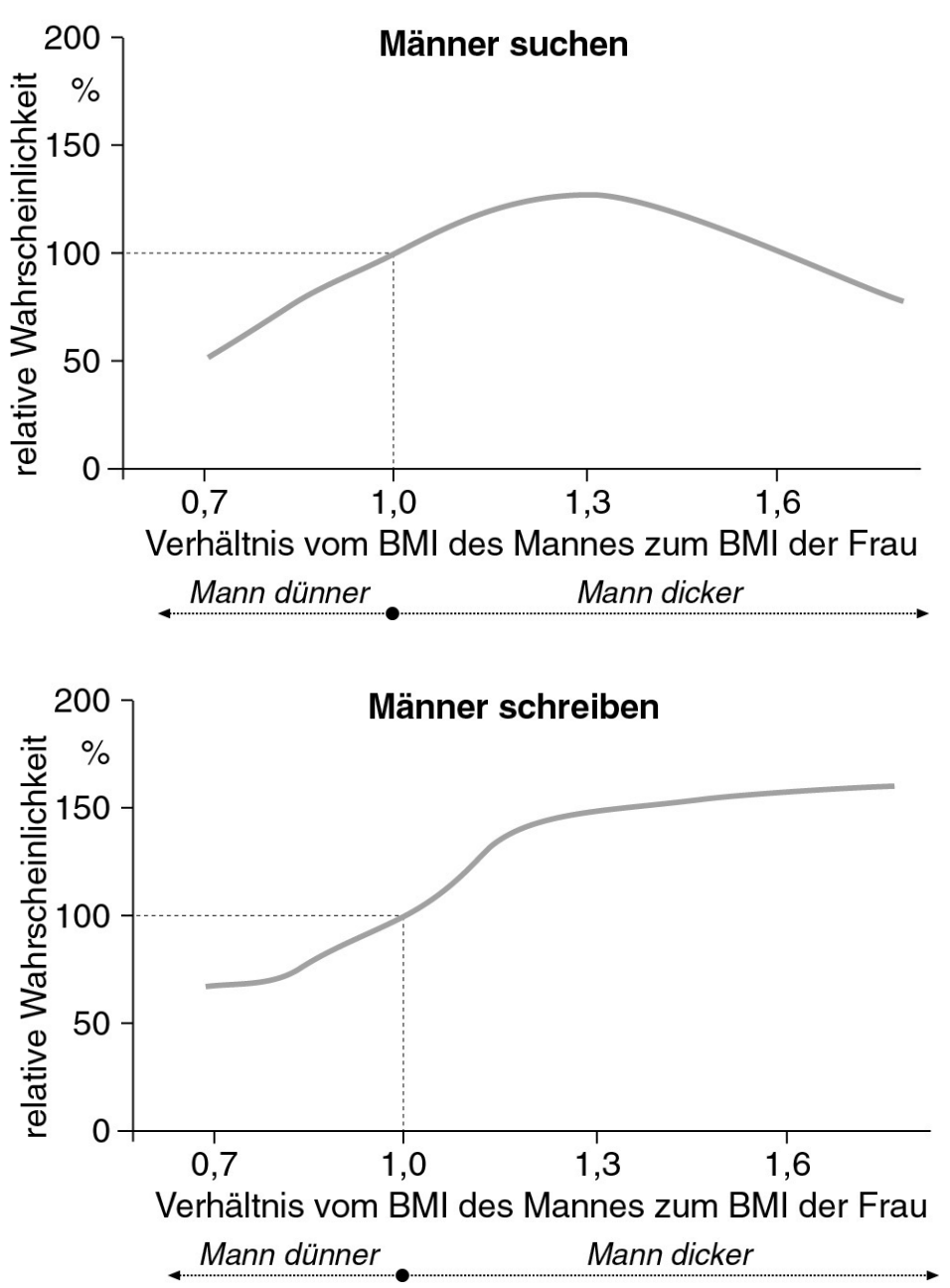

Abb. 4 Rolle des Körpergewichts (BMI) beim Dating. Oben: Wen Männer suchen. Relative Wahrscheinlichkeit (bezogen auf die Wahrscheinlichkeit bei gleichem BMI), in die Suche eines Mannes eingeschlossen zu werden in Abhängigkeit vom Verhältnis des BMI des (suchenden) Mannes und der (potenziellen) Partnerin. Unten:Wem Männer schreiben. Relative Wahrscheinlichkeit, dass ein Mann einer Frau schreibt in Abhängigkeit vom Verhältnis seines BMI zu dem der potenziellen Partnerin (stark schematisierte Darstellung nach 1, Fig. 4 A, B).

und Entscheidungsschritt. Bei ihrer Suche bevorzugen Männer solche Frauen, deren BMI sich zu dem des Mannes wie 1: 1,3 verhält, also schlanker sind als sie selbst, aber nicht zu viel schlanker ( Abb. 4 links). Wenn es jedoch darum geht, ob man(n) einer Frau schreibt, dann scheint der vielzitierte Satz, eine Frau könne nie zu dünn sein, zu gelten ( $\triangle$ Abb. 4 rechts).

Weitere interessante Befunde waren kurz zusammengefasst: Kein Foto von sich zu senden, lässt die eigene Chance, von jemandem ausgesucht zu werden, um das 20-Fache sinken, egal, ob man ein Mann oder eine Frau ist. Auch Raucher haben es, unabhängig vom Geschlecht, schwer: Ihre Chance, in den Suchraum zu gelangen, ist im Vergleich zu Nichtrauchern um das 10-Fache geringer.

\section{Obwohl es Damenfußball und} männliche Models gibt, sind die Boulevardblätter voll von Beziehungen zwischen zierlichen jungen Frauen und muskulösen Fußballern.

Bemerkenswert erscheint zudem die extreme Variabilität der Verhaltensweisen. So lassen sich bestimmte „Typen“ identifizieren, beispielsweise korpulente Männer um die 40 , die eine deutlich jüngere und vor allem schlankere Frau bevorzugen; oder Frauen um die 40, die nach einen deutlich älteren Mann Ausschau halten. Paarungen dieser Art sind also eher kein Zufall, sondern passen auf bestimmte biologische und kulturelle "Anforderungsprofile“ bei der wechselseitigen Auswahl. Es kann bei der Partnerwahl offensichtlich manchmal ganz ähnlich sein wie beim Blinden und beim Lahmen: Unterschiede tun sich zusammen und haben davon im konkreten Lebensvollzug beide einen Vorteil.

Insgesamt passen die Ergebnisse der Studie sehr gut zu den bekannten Studien zum Verhalten von Menschen bei der Partnerwahl: Dachte man früher vor allem an psychologische und soziologische Gesichtspunkte im Sinne einer kulturellen Beeinflussung, so setzte sich in den vergangenen knapp drei Jahrzehnten die Erkenntnis durch, dass sehr vieles, was zunächst kulturell höchst variable scheint, im Grunde eindeutige biologische Wurzeln hat und in seiner Homogenität schon fast als "langweilig“ zu bezeichnen ist: Männer sind Männer und Frauen sind Frauen - überall!

So fand David Buss (2) in einer Untersuchung von 10047 Personen aus 33 Ländern auf allen sechs Kontinenten und fünf Inseln erstaunliche Gemeinsamkeiten trotz aller Unterschiede der Kulturen - im Hinblick auf die der Partnerwahl zugrunde liegenden Prinzipien. Zwar gibt es kulturelle Unterschiede - Keuschheit vor der Ehe ist in Taiwan, Indien und im Iran für Männer und Frauen ein unabdingbares Kriterium, spielt hingegen in Finnland, Frankreich, Dänemark, Schweden oder Deutschland kaum eine Rolle (4).

Wenn es jedoch darum geht, wie wichtig materielle Ressourcen sind, so sind diese den Frauen überall auf der Welt wichtiger als den Männern, und Männer legen wiederum überall auf der Welt mehr Wert auf Schönheit (ein Zeichen von Fruchtbarkeit) als Frauen. Beides erklärt übrigens, warum - fast - überall auf der Welt bei Paaren ein Altersunterschied besteht (Frauen jünger, Männer älter), dessen Ausmaß allerdings von kulturellen Rahmenbedingungen abhängt: Wo Armut 
herrscht, sind materielle Ressourcen und wo es keine Altersversorgung gibt, sind Nachkommen vergleichsweise wichtiger. Dies erklärt, warum in afrikanischen Ländern der Altersunterschied von Paaren bei der Hochzeit weltweit am größten ist. Und es erklärt auch die einzige Ausnahme Schweden. Dort sind die Männer bei der Hochzeit im Mittel ein klein bisschen jünger als die Frauen. Kein Wunder: Wenn der Staat die (Alters-)Versorgung weitgehend übernommen hat, entfällt der Versorgungsaspekt.

Auch Kenrick und Keefe (7) fanden in einem methodisch und im Hinblick auf die untersuchten Stichproben sehr variantenreichen Ansatz, dass der Altersunterschied überall zu finden ist, egal, wo und wie man schaut: Man findet ihn in Heiratsanzeigen (ganz normaler sowie sehr reicher Leute) und Heiratsstatistiken in den USA, Europa und Indien, heute und auch vor fast hundert Jahren, wie sechs sehr unterschiedliche Studien zeigten.

Auch die Ergebnisse einer ältern Studie zum Online-Dating bei knapp 12000 Personen aus San Diego und Boston bestätigen dies: „Wir finden in einem typischen Paar [das sich gefunden hat], dass die Männer 1,3 Jahre älter und 5,9 Inch größer sind, einen höheren BMI haben und schlechter aussehen als die Frauen. Sie verdienen 49000 Doller [pro Jahr] mehr und haben ein halbes Jahr Ausbildung mehr" (6, Übersetzung durch den Autor).

Diese Studien zeigen, dass die uns umgebende digitale Welt eines nicht verändert: uns selbst.

Bliebe noch eine weitere sehr große, USamerikanische Studie zum Verhalten (von jeweils heterosexuellen 528800 Männern und 405021 Frauen) in sozialen OnlineMedien zu erwähnen, in der sich vor allem eines zeigte: die ethnische Zugehörigkeit als k.o.-Kriterium: Amerikaner mit afrikanischer Abstammung haben es auf dem amerikanischen Heiratsmarkt schwer. ${ }^{4}$

Insgesamt zeigen diese Studien auf eindrucksvolle Weise und anhand sehr großer Datenmengen, dass die uns umgebende digitale Welt eines nicht verändert: uns selbst. Wie schon die kürzlich an dieser Stelle diskutierten Daten zum Telefonieren (ca. eine halbe Milliarde Telefonate betreffend) zeigten (13), verhalten sich die Menschen angesichts der neuen Kommunikationsmöglichkeiten keineswegs anders als vor 100000 Jahren, sondern genau gleich. Das ist zunächst einmal beruhigend, denn es geht bei den untersuchten Verhaltensweisen ja um nichts weniger als um solche, die den Fortbestand der Menschheit sichern. Wir ändern uns also nicht, obwohl dies durchaus möglich wäre. Wenn sich in den USA „60\% aller verheirateten Paare in der Schule, bei der Arbeit, bei einer privaten Party, in der Kirche oder in einem Sport- oder Freizeitclub kennengelernt haben" (6, Übersetzung durch den Autor), dann könnte man erwarten, das die Möglichkeiten des Online-Treffens den Zufall aus solchen Begegnungen zugunsten von bedeutsamen Variablen verdrängen könnten. Eine davon könnte Bildung sein. Macht also das Online-Dating künftige Beziehungen zufriedenstellender und damit „haltbarer"? Die Antwort der Empirie auf diese Frage ist „eher nicht“. Denn gerade die Tatsache, dass manche Variablen als k.o.-Kriterien ganz zu Beginn des Suchprozesses eingesetzt werden, spricht gegen den Gedanken, die Digitalisierung des Heiratsmarktes ermögliche eine Zunahme von dessen Nuancierung: „Does education, an increasingly important achieved status, trump racial preferences in the dating market? The answer is a resounding no [...]. White men and women with a college degree are more likely to contact and reciprocate to white daters without a college degree over black daters with a college degree" (8). Daraus ergibt sich die zunächst kontraintuitive Konsequenz, dass der Bildungsstand durch Online-Dating für die Paarbildung an Bedeutung verliert, wie auch Hitsch und Mitarbeiter bereits zuvor

4 Im Originaltext: „When Internet daters search for potential mates, they are most likely to approach those who have the same racial identity. Yet a salient racial hierarchy dominates in the reciprocating process. White men's and women's messages are likely to be reciprocated by daters of other groups, but white women reciprocate mostly only to white men. Black daters, particularly black women, tend to be ignored when they contact nonblack groups, even though they respond to out-groups no less frequently. Asian and Hispanic daters seem to be at the middle of the racial hierarchy" (8). aufgrund ähnlicher Beobachtungen feststellten: „An interesting consequence, therefore, is that sorting along education could decrease if online dating became a common means of finding a partner" (6, Übersetzung und Hervorhebung durch den Autor). Hierzu nochmals Lin und Lundquist (8, S. 207): „Importantly, we find that education does not mediate the observed racial preferences among white men and women. White men and women with a college degree prefer to contact and reciprocate to white daters without a college degree over black daters with a college degree“.

Auch wenn also die Menschen so sind und auch digital so bleiben, wie sie sind, bedeutet dies nicht, dass die Digitalisierung aller Aspekte unseres Lebens, einschließlich unseres Sexuallebens, keine Spuren hinterlassen würde. Nur sind es vielleicht andere als man dies erwarten würde. Und ob durch sie alles besser wird, darf man(n) bezweifeln, frau auch.

\section{Literatur}

1. Bruch E, Feinberg F, Lee KY. Extracting multistage screening rules from online dating activity data. PNAS 2016; August 30, published ahead of print.

2. Buss DM. Sex differences in human mate preferences: Evolutionary hypotheses testing in $37 \mathrm{cul}-$ tures. Behavioral and Brain Sciences 1989; 12: $1-49$.

3. Buss DM. The evolution of desire: Strategies of human mating. New York: Basic Books 1994/2003.

4. Buss Dt. The Evolution of human mating. Acta Psychologica Sinica 2007; 39: 502-512.

5. Cowan N. The magical mystery four: How is working memory capacity limited, and why? Curr Dir Psychol Sci 2010; 19: 51-57.

6. Hitsch G, Hortaçsu A, Ariely D. Matching and sorting in online dating. Am Econ Rev 2010; 100: 130-63.

7. Kenrick DT, Keefe RC. Age preferences in mates reflect sex differences in human reproductive strategies. Behavioral and Brain Sciences 1992; 15: 75-133.

8. Lin K, Lundquist J. Mate selection in cyberspace. Am J Sociology 2013; 119: 183-215.

9. Miller GA. The magical number seven, plus or minus two: Some limits on our capacity for processing information. Psychological Review 1956; 63: 81-97.

10. Spitzer M. Entscheiden. Im Rahmen von Bernoulli zur Amygdala. Nervenheilkunde 2006; 25: 969-73.

11. Spitzer M. Die Qual der Wahl. Nervenheilkunde 2007; 26: 810-12.

12. Spitzer M. Die Wissenschaft vom Flirten. Nervenheilkunde 2011; 30: 855-62.

13. Spitzer M. Telefonieren studieren. Wer, mit wem, wie oft und wie viele? Nervenheilkunde 2016; 35: 371-74. 